\title{
High-Performance Up-and-Downdating via Householder-like Transformations
}

\author{
ROBERT A. VAN DE GEIJN \\ The University of Texas at Austin \\ and \\ FIELD G. VAN ZEE \\ The University of Texas at Austin
}

\begin{abstract}
We present high-performance algorithms for up-and-downdating a Cholesky factor or QR factorization. The method uses Householder-like transformations, sometimes called hyperbolic Householder transformations, that are accumulated so that most computation can be cast in terms of high-performance matrix-matrix operations. The resulting algorithms can then be used as building blocks for an algorithm-by-blocks that allows computation to be conveniently scheduled to multithreaded architectures like multicore processors. Performance is shown to be similar to that achieved by a blocked QR factorization via Householder transformations.
\end{abstract}

Categories and Subject Descriptors: G.4 [Mathematical Software]: —Efficiency

General Terms: Algorithms; Performance

Additional Key Words and Phrases: linear algebra, libraries, high-performance

\section{INTRODUCTION}

Consider the Linear Least-Squares problem that, given a matrix $A \in \mathbb{C}^{m \times n}$ with linearly independent columns and $y \in \mathbb{C}^{m}$, computes $x \in \mathbb{C}^{n}$ that minimizes $\| A x-$ $y \|_{2}$. This problem is typically solved via one of two methods:

Method of Normal Equations: Solve $A^{H} A x=A^{H} y$ by computing the Cholesky factor of $A^{H} A$, upper triangular matrix $R$, followed by forward and backward substitution to solve $R^{H} R x=A^{H} y$.

QR factorization (via Householder transformations): Compute the $Q R$ factorization $A=Q R$ where $Q$ is an orthogonal $m \times n$ matrix and $R$ is an upper triangular $n \times n$ matrix. Solve $R x=Q^{H} y$.

In this paper, we concern ourselves with the following prototypical scenario: Let rows of the appended system $(A \mid y)$ represent observations that have been taken, for example, over time. These observations can be partitioned into three groups:

Authors' addresses: Robert A. van de Geijn and Field G. Van Zee, Department of Computer Science, The University of Texas at Austin, Austin, TX 78712, \{rvdg,field\}@cs.utexas.edu.

Permission to make digital/hard copy of all or part of this material without fee for personal or classroom use provided that the copies are not made or distributed for profit or commercial advantage, the ACM copyright/server notice, the title of the publication, and its date appear, and notice is given that copying is by permission of the ACM, Inc. To copy otherwise, to republish, to post on servers, or to redistribute to lists requires prior specific permission and/or a fee.

(C) $20 \mathrm{YY}$ ACM 0098-3500/20YY/1200-0001 $\$ 5.00$ 
$(A \mid y)=\left(\begin{array}{c|c}B \mid b \\ \overline{\bar{C}} \mid c \\ \hline \bar{D} \mid d\end{array}\right)$ where the Cholesky factor corresponding to $\left(\frac{B}{\bar{D}}\right)$ has already been computed: $B^{H} B+D^{H} D=R^{H} R$. Now, the rows of $D$ represent old data that we would like to remove while the rows of $C$ represent new data that we would like to add to the Linear Least-Squares problem. Thus, we would like to compute the Cholesky factor corresponding to $\left(\frac{B}{\bar{C}}\right)$ leveraging the already computed $R$. The right-hand side has to be updated correspondingly, which is discussed in Section 6 .

In [Stewart and Stewart 1998] hyperbolic Householder transformations are reviewed for this problem and analyzed both from an algorithmic and numerical stability point of view. In that paper, references to the literature can also be found. The present paper builds on the insights in that paper and combines it with insights from other papers [Bischof and Van Loan 1987; Schreiber and Van Loan 1989; Joffrain et al. 2006; Walker 1988; Puglisi 1992; Yan and Chung 1997; Sun 1996] that focus on aggregrating multiple Householder-like transformations into a block transformation. The contribution of the present paper is a practical high-performance algorithm for up- and/or downdating that can be implemented as a library routine using the level-3 BLAS [Dongarra et al. 1990].

The remainder of the paper is structured as follows. In Section 2 we discuss a family of Householder-like transformations and how to accumulate them into a block transformation. Updating and downdating are discussed separately in Sections 3 and 4, respectively, and then combined in Section 5 in which a blocked algorithm is also given. How to use an up- and/or downdated system to solve the new Linear Least-Squares problem is discussed in Section 6. A brief overview of the algorithmby-blocks concept is given in Section 7. Performance is reported in Section 8 and concluding remarks can be found in the final section.

\section{A FAMILY OF HOUSEHOLDER TRANSFORMATIONS}

In the following discussion, we will let $\Sigma \in \mathbb{R}^{n \times n}$ with $\Sigma=\operatorname{diag}(1, \pm 1, \cdots, \pm 1)$ so that $\Sigma \Sigma=I .{ }^{1}$ Such a matrix is referred to as a signature matrix. We make the choice that the first diagonal element equals one so as to simplify our discussion. Then, by design, $\Sigma e_{0}=e_{0}$, where $e_{0}$ is the first column of the identity matrix.

TheOREm 1. Let $w \in \mathbb{C}^{n}$ and $\tau=w^{H} \Sigma w / 2 \neq 0$. Then

$$
\left(I-\frac{1}{\tau} \Sigma w w^{H}\right) \Sigma\left(I-\frac{1}{\tau} \Sigma w w^{H}\right)^{H}=\Sigma .
$$

Proof: Under the assumptions of the theorem

$$
\left(I-\frac{1}{\tau} \Sigma w w^{H}\right) \Sigma\left(I-\frac{1}{\tau} \Sigma w w^{H}\right)^{H}=\Sigma-2 \frac{1}{\tau} \Sigma w w^{H} \Sigma+\frac{2}{\tau} \Sigma w w^{H} \Sigma=\Sigma .
$$

endofproof

\footnotetext{
${ }^{1}$ Here, $\operatorname{diag}\left(\sigma_{0}, \sigma_{1}, \cdots, \sigma_{n-1}\right)$ returns a diagonal matrix whose entries are $\sigma_{0}, \sigma_{1}, \cdots, \sigma_{n-1}$.

ACM Transactions on Mathematical Software, Vol. V, No. N, Month 20YY.
} 
When $\Sigma=I$ the $\left(I-\frac{1}{\tau} \Sigma w w^{H}\right)$ in the above theorem is the traditional Householder transformation or reflector. If $\Sigma=\left(\begin{array}{l||l}I \| & 0 \\ \hline \overline{0 \|-I}\end{array}\right)$ it is referred to as a hyperbolic Householder Transformation.

Theorem 2. Let $x \in \mathbb{C}^{n}, \chi_{0}=e_{0}^{T} x$ be its first element, $\lambda$ be chosen such that $|\lambda|^{2}=x^{H} \Sigma x$ and $\bar{\lambda} \chi_{0}\left(=\bar{\chi}_{0} \lambda\right)$ is real, $w=x+\lambda e_{0}$, and $\tau=\frac{w^{H} \Sigma w}{2} \neq 0$. Then $\left(I-\frac{1}{\tau} \Sigma w w^{H}\right)^{H} x=-\lambda e_{0}$.

Proof: Under the assumptions of the theorem

$$
\frac{2 w^{H} \Sigma x}{w^{H} \Sigma w}=\frac{2\left(x+\lambda e_{0}\right)^{H} \Sigma x}{\left(x+\lambda e_{0}\right)^{H} \Sigma\left(x+\lambda e_{0}\right)}=\frac{2\left(x^{H} \Sigma x+\bar{\lambda} \chi_{0}\right)}{x^{H} \Sigma x+2 \bar{\lambda} \chi_{0}+|\lambda|^{2}}=1
$$

and

$$
\left(I-\frac{1}{\tau} \Sigma w w^{H}\right)^{H} x=x-\frac{1}{\tau} w w^{H} \Sigma x=x-\frac{2 w^{H} \Sigma x}{w^{H} \Sigma w}\left(x+\lambda e_{0}\right)=-\lambda e_{0} .
$$

COrollary 3. In Thm. 2, if $\chi_{0}$ is real, then $\lambda= \pm \sqrt{x^{H} \Sigma x}$.

endofproof

The Cholesky factor $R$ that is being updated and/or downdated often has real diagonal elements, each of which takes the form of $\chi_{0}$ in the vector $x$ of Theorem 2 . The vector $w$ can be normalized by dividing by a nonzero scalar, in which case the following steps provide a robust way of computing $w$ and $\lambda$ so that $w$ has a unit first element:

$-\lambda:=\operatorname{sign}\left(\chi_{0}\right) \sqrt{x^{H} \Sigma x} . w:=x+\lambda e_{0}$. (Note: the choice of the sign means that $\lambda$ and $\chi_{0}$ have the same sign, thus avoiding catastrophic cancellation that can lead to unnecessary numerical inaccuracy).

—If $\omega_{0}=e_{0}^{T} w$ equals zero, then $w:=e_{0}$ else $w:=w / \omega_{0}$. (This step normalizes $w$ so that it has a unit first element.)

Definition 4. Let $x \in \mathbb{C}^{n}$ be such that $x^{H} \Sigma x \neq 0$. We define the function ${ }^{2}$

$$
\left[\tilde{\chi}_{0}, w_{1}, \tau\right]:=\text { GeneralHouse }\left(\Sigma, \chi_{0}, x_{1}\right)
$$

so that $\left(I-\frac{1}{\tau} \Sigma w w^{H}\right)^{H} x=\tilde{\chi}_{0} e_{0}$, where $x=\left(\frac{\chi_{0}}{x_{1}}\right), w=\left(\frac{1}{w_{1}}\right)$, and $\tau=\frac{w^{H} \Sigma w}{2}$.

TheOREM 5. Let the matrix $W_{k-1} \in \mathbb{C}^{n \times k}$ have linearly independent columns. Partition $W_{k-1}$ by columns as

$$
W_{k-1}=\left(w_{0}\left|w_{1}\right| \cdots \mid w_{k-1}\right)
$$

and let $\tau_{i} \neq 0,0 \leq i<k$. Then for $0 \leq j<k$ there exists a $j \times j$ nonsingular upper triangular matrix $T_{j-1}$ such that

$$
\left(I-\frac{1}{\tau_{0}} \Sigma w_{0} w_{0}^{H}\right) \cdots\left(I-\frac{1}{\tau_{j-1}} \Sigma w_{j-1} w_{j-1}^{H}\right)=\left(I-\Sigma W_{j-1} T_{j-1}^{-1} W_{j-1}^{H}\right)
$$

${ }^{2}$ Throughout this paper we will present functions which take the form [output-list] := Func (input-list). In this syntax, the function FunC takes the values in input-list as input arguments and outputs the values in output-list. Later on, when we show algorithms which use these functions, some parameters may appear in both the input-list and output-list, which indicates that the value is read and then overwritten. 
The matrices $T_{j}$ are given by the recurrence $T_{0}=\tau_{0}$ and $T_{j}=\left(\begin{array}{c|c}T_{j-1} & W_{j-1}^{H} \Sigma w_{j} \\ \hline 0 & \tau_{j}\end{array}\right)$ for $1 \leq j<k$.

Proof: Proof by induction on $j$.

Base case. $j=0$ : Trivially true.

Inductive step. Induction Hypothesis (I.H.): Assume that

$$
\left(I-\frac{1}{\tau_{0}} \Sigma w_{0} w_{0}^{H}\right) \cdots\left(I-\frac{1}{\tau_{j-1}} \Sigma w_{j-1} w_{j-1}^{H}\right)=I-\Sigma W_{j-1} T_{j-1}^{-1} W_{j-1}^{H} .
$$

We need to show that

$$
\left(I-\frac{1}{\tau_{0}} \Sigma w_{0} w_{0}^{H}\right) \cdots\left(I-\frac{1}{\tau_{j-1}} \Sigma w_{j-1} w_{j-1}^{H}\right)\left(I-\frac{1}{\tau_{j}} \Sigma w_{j} w_{j}^{H}\right)=I-\Sigma W_{j} T_{j}^{-1} W_{j}^{H} .
$$

But

$$
\begin{aligned}
& \left(I-\frac{1}{\tau_{0}} \Sigma w_{0} w_{0}^{H}\right) \cdots\left(I-\frac{1}{\tau_{j}} \Sigma w_{j} w_{j}^{H}\right)=\left(I-\Sigma W_{j-1} T_{j-1}^{-1} W_{j-1}^{H}\right)\left(I-\frac{\Sigma w_{j} w_{j}^{H}}{\tau_{j}}\right) \\
& =I-\Sigma\left(W_{j-1} \mid w_{j}\right)\left(\begin{array}{c|c}
T_{j-1}^{-1} & -T_{j-1}^{-1} W_{j-1}^{H} \Sigma w_{j} / \tau_{j} \\
\hline 0 & 1 / \tau_{j}
\end{array}\right)\left(W_{j-1} \mid w_{j}\right)^{H} \\
& =I-\Sigma\left(W_{j-1} \mid w_{j}\right)\left(\begin{array}{c|c}
T_{j-1} & W_{j-1}^{H} \Sigma w_{j} \\
\hline 0 & \tau_{j}
\end{array}\right)^{-1}\left(W_{j-1} \mid w_{j}\right)^{H}=I-\Sigma W_{j} T_{j}^{-1} W_{j}^{H} .
\end{aligned}
$$

By the Principle of Mathematical Induction the desired result holds.

endofproof

TheOREM 6. Let $W \in \mathbb{C}^{n \times k}$ be a matrix with linearly independent columns such that $W^{H} \Sigma W$ has nonzero diagonal elements. Then there exists a unique nonsingular upper triangular matrix with real diagonal elements $T \in \mathbb{C}^{k \times k}$ such that $\left(I-\Sigma W T^{-1} W^{H}\right) \Sigma\left(I-\Sigma W T^{-1} W^{H}\right)^{H}=\Sigma$. This matrix $T$ satisfies $T+T^{H}=$ $W^{H} \Sigma W$ so that $T=\operatorname{striu}\left(W^{H} \Sigma W\right)+\frac{1}{2} \operatorname{diag}\left(W^{H} \Sigma W\right) .^{3}$

Proof: Theorem 5 provides a proof of existence. (The $w_{i}$ and $\tau_{i}$ 's in that theorem equal the columns of $W$ and diagonal elements of $W^{T} \Sigma W$, respectively.) Now,

$$
\begin{aligned}
\Sigma & =\left(I-\Sigma W T^{-1} W^{H}\right) \Sigma\left(I-\Sigma W T^{-1} W^{H}\right)^{H} \\
& =\Sigma-\Sigma W T^{-1} W^{H} \Sigma-\Sigma W T^{-H} W^{H} \Sigma+\Sigma W T^{-1} W^{H} \Sigma W T^{-H} W^{H} \Sigma
\end{aligned}
$$

so that $0=\Sigma W\left(T^{-1}+T^{-H}-T^{-1} W^{H} \Sigma W T^{-H}\right) W^{H} \Sigma$. Since $\Sigma W$ is nonsingular we find that $0=T^{-1}+T^{-H}-T^{-1} W^{H} \Sigma W T^{-H}$ from which we conclude that $T^{H}+T=W^{H} \Sigma W$.

Because $T$ is upper triangular and has real valued diagonal elements, $T=$ $\operatorname{striu}\left(W^{H} \Sigma W\right)+\frac{1}{2} \operatorname{diag}\left(W^{H} \Sigma W\right)$.

endofproof

\footnotetext{
${ }^{3}$ Note that $\operatorname{striu}(A)$ returns a matrix consisting of the strictly upper triangular part of $A$ with zeros elsewhere. Also, in this context, $\operatorname{diag}(A)$ returns the matrix consisting of the diagonal of $A$ with zeros elsewhere.
}

ACM Transactions on Mathematical Software, Vol. V, No. N, Month 20YY. 


\section{UPDATING}

Let us consider $A=\left(\frac{B}{\bar{C}}\right)$ with $A^{H} A=B^{H} B+C^{H} C=\tilde{R}^{H} \tilde{R}$, where $\tilde{R}$ is the Cholesky factor of $A^{H} A$. Here we will assume that $A$ has linearly independent columns. The question is whether, if we know that the Cholesky factor of $B^{H} B$ is $R$, we can cheaply compute the Cholesky factor of $A^{H} A$. This is known as the updating problem. We know that $\tilde{R}^{H} \tilde{R}=A^{H} A=B^{H} B+C^{H} C=R^{H} R+C^{H} C$. We also know that if $B=Q R$ is a QR factorization of $B$ and $\left(\frac{R}{\bar{C}}\right)=\hat{Q} \hat{R}$, then

$$
A=\left(\frac{B}{\bar{C}}\right)=\left(\begin{array}{c}
Q R \\
\hline \bar{C}
\end{array}\right)=\left(\begin{array}{c|c}
Q & 0 \\
\hline \overline{0} \| I
\end{array}\right)\left(\frac{R}{\bar{C}}\right)=\left(\begin{array}{c|c}
Q & 0 \\
\hline \overline{0} \| I
\end{array}\right) \hat{Q} \hat{R}=\check{Q} \hat{R}
$$

so that $A=\check{Q} \hat{R}$ is a $\mathrm{QR}$ factorization of $A$. Because of the uniqueness of the QR factorization (modulo signs), $\hat{R}=\tilde{R}$. This then provides us with the desired Cholesky factor. We conclude that to compute $\tilde{R}$ it suffices to compute the QR factorization of $\left(\frac{R}{\bar{C}}\right)$.

A stable way for computing the QR factorization of $\left(\frac{R}{\bar{C}}\right)$ relies on Householder transformations: given matrix $\left(\frac{R}{\bar{C}}\right)$ where $R$ is an $n \times n$ upper triangular matrix and $C \in \mathbb{C}^{m_{C} \times n}$, we would like to compute $\left\{H_{0}, \cdots, H_{n-1}\right\}$ so that

$H_{n-1}^{H} \cdots H_{0}^{H}\left(\frac{R}{\bar{C}}\right)=\left(\frac{\tilde{R}}{\overline{0}}\right) \quad$ and $\quad H_{j}^{H}=H_{j}=I-\frac{1}{\tau_{j}} u_{j} u_{j}^{H}$ with $\tau_{j}=u_{j}^{H} u_{j} / 2$.

We recognize this as the special case of the Generalized Householder Transformation where $\Sigma=I$, in other words, the classical Householder Transformation.

Definition 7. Let $x=\left(\frac{\chi_{0}}{x_{1}}\right) \in \mathbb{C}^{n}$ with $\chi_{0} \in \mathbb{R}$ be such that $\|x\|_{2} \neq 0$. We define the function

$$
\left[\tilde{\chi_{0}}, u_{1}, \tau\right]:=\operatorname{House}\left(\chi_{0}, x_{1}\right)
$$

so that $\left(\left(\begin{array}{c|c}1 & 0 \\ \hline 0 & I\end{array}\right)-\frac{1}{\tau}\left(\frac{1}{u_{1}}\right)\left(\frac{1}{u_{1}}\right)^{H}\right)\left(\frac{\chi_{0}}{x_{1}}\right)=\left(\frac{\hat{\chi}_{0}}{0}\right)$, where $\tau=\frac{1+u_{1}^{H} u_{1}}{2}$.

Given the function House, we now wish to construct an algorithm that, given the Cholesky factor $R$ of $A^{H} A$, computes the Cholesky factor of $A^{H} A+C^{H} C$. Here is the basic idea: Assume that the computation has progressed so that the matrices contain

$$
\left(\frac{R}{\bar{C}}\right)=\left(\begin{array}{c|c|c}
R_{00} & r_{01} & R_{02} \\
\hline 0 & \rho & r_{12}^{T} \\
\hline 0 & 0 & R_{22} \\
\hline \hline 0 & c_{1} & C_{2}
\end{array}\right) .
$$




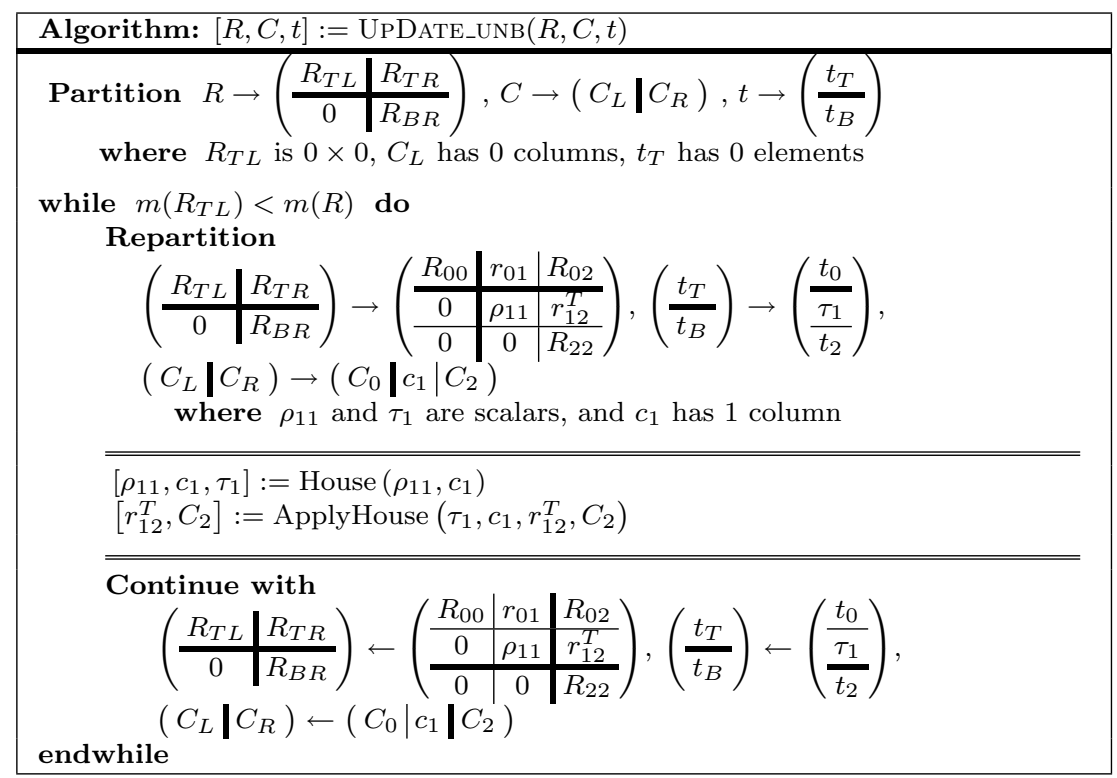

Fig. 1. Unblocked algorithm for updating.

In the current step of the algorithm, a Householder transformation is computed and applied so that

$$
\left.\left[\begin{array}{c|c|c|c}
I & 0 & 0 & 0 \\
\hline 0 & 1 & 0 & 0 \\
\hline 0 & 0 & I & 0 \\
\hline 0 & 0 & 0 & I
\end{array}\right)-\frac{1}{\tau}\left(\begin{array}{c}
0 \\
\hline \frac{1}{0} \\
\overline{\overline{u_{1}}}
\end{array}\right)\left(\begin{array}{c}
0 \\
\hline 1 \\
\hline 0 \\
\hline \overline{u_{1}}
\end{array}\right)\right]\left(\begin{array}{c|c|c|c|c}
R_{00} & r_{01} & R_{02} \\
\hline 0 & \rho & r_{12}^{T} \\
\hline 0 & 0 & R_{22} \\
\hline 0 & c_{1} & C_{2}
\end{array}\right)=\left(\begin{array}{c|c|c|c}
R_{00} & r_{01} & R_{02} \\
\hline 0 & \tilde{\rho} & \tilde{r}_{12}^{T} \\
\hline 0 & 0 & R_{22} \\
\hline 0 & 0 & \tilde{C}_{2}
\end{array}\right) .
$$

Since the Householder transformation associated with $u_{1}$ is formulated to annihilate the vector $c_{1}$, the algorithm may simply set $c_{1}$ to zero (or, more practically, it may overwrite $c_{1}$ with $u_{1}$ to reduce storage). However, we must then use $\tau$ and $u_{1}$ to apply the Householder transformation to $r_{12}^{T}$ and $C_{2}$. Let us define a function ApplyHouse which serves this purpose:

$$
\left[\tilde{r}_{12}^{T}, \tilde{C}_{2}\right]:=\operatorname{ApplyHouse}\left(\tau_{1}, u_{1}, r_{12}^{T}, C_{2}\right) .
$$

An algorithm based on these steps is given in Figure 1.

\section{DOWNDATING}

Now, let us consider the alternative problem where $A=\left(\frac{B}{\bar{D}}\right)$ with $A^{H} A=$ $B^{H} B+D^{H} D=R^{H} R$, where $D \in \mathbb{C}^{m_{D} \times n}$ and $R \in \mathbb{C}^{n \times n}$ is the Cholesky factor of $A^{H} A$. The new question becomes how to compute the Cholesky factor of $B^{H} B$ from $R$ and $D$. This is known as the downdating problem. Let us call the desired ACM Transactions on Mathematical Software, Vol. V, No. N, Month 20YY. 
Cholesky factor $\tilde{R}$. We know that

$$
\tilde{R}^{H} \tilde{R}=B^{H} B=R^{H} R-D^{H} D=\left(\frac{R}{\bar{D}}\right)^{H}\left(\begin{array}{c||c}
I_{n} & 0 \\
\hline \hline 0 & -I_{m_{D}}
\end{array}\right)\left(\begin{array}{c}
R \\
\hline \bar{D}
\end{array}\right)
$$

In the remainder of this section, we will define

$$
\Sigma_{n, m}=\left(\begin{array}{c||c}
I_{n} & 0 \\
\hline \hline 0 & -I_{m}
\end{array}\right) .
$$

The goal is going to be to compute a sequence of transformations, $\left\{S_{0}, S_{1}, \cdots, \mathbb{S}_{n-1}\right\}$ such that

$$
S_{j} \Sigma_{n, m_{D}} S_{j}^{H}=\Sigma_{n, m_{D}}
$$

and

$$
\begin{aligned}
& B^{H} B=\left(\begin{array}{c}
R \\
\hline \bar{D}
\end{array}\right)^{H}\left(\begin{array}{c||c}
I_{n} & 0 \\
\hline \hline 0 & -I_{m_{D}}
\end{array}\right)\left(\begin{array}{c}
R \\
\hline \bar{D}
\end{array}\right) \\
& =\left(\frac{R}{\bar{D}}\right)^{H} S_{0} \cdots S_{n-1} \Sigma_{n, m_{D}} S_{n-1}^{H} \cdots S_{0}^{H}\left(\frac{R}{\bar{D}}\right) \\
& =\left(\frac{\tilde{R}}{\overline{0}}\right)^{H} \Sigma_{n, m_{D}}\left(\frac{\tilde{R}}{\overline{0}}\right)=\tilde{R}^{H} \tilde{R}
\end{aligned}
$$

In other words, given matrix $\left(\frac{R}{\bar{D}}\right)$ where $R$ is an $n \times n$ upper triangular matrix, we would like to compute $\left\{S_{0}, \cdots, S_{n-1}\right\}$ so that $S_{n-1}^{H} \cdots S_{0}^{H}\left(\frac{R}{\bar{D}}\right)=\left(\frac{\tilde{R}}{\overline{0}}\right)$ and $S_{j} \Sigma_{n, m_{D}} S_{j}=\Sigma_{n, m_{D}}^{H}$.

DeFinition 8. Let $x=\left(\frac{\chi_{0}}{x_{1}}\right) \in \mathbb{C}^{n}$ with $\chi_{0} \in \mathbb{R}$ be such that $x^{H} \Sigma_{1, n-1} x=$ $\left|\chi_{0}\right|^{2}-x_{1}^{H} x_{1} \neq 0$. We define the function

$$
\left[\tilde{\chi}_{0}, v_{1}, \tau\right]:=\operatorname{HHouse}\left(\chi_{0}, x_{1}\right)
$$

so that $\left(\left(\begin{array}{c|c}1 & 0 \\ \hline 0 & I\end{array}\right)-\frac{1}{\tau}\left(\begin{array}{c|c}1 & 0 \\ \hline 0 & -I\end{array}\right)\left(\frac{1}{v_{1}}\right)\left(\frac{1}{v_{1}}\right)^{H}\right)\left(\frac{\chi_{0}}{x_{1}}\right)=\left(\begin{array}{c}\hat{\chi}_{0} \\ \hline 0\end{array}\right)$, where $\tau=$ $\frac{1-v_{1}^{H} v_{1}}{2}$.

We recognize this as the special case of the Generalized Householder Transformation where $\Sigma=\Sigma_{1, n-1}$. This special case is referred to as a hyperbolic Householder Transformation in the literature.

Given the function HHouse, we now wish to construct an algorithm that, given the Cholesky factor $R$ of $A^{H} A$, computes the Cholesky factor of $A^{H} A-D^{H} D$. Here is the basic idea: Assume that the computation has progressed so that the 


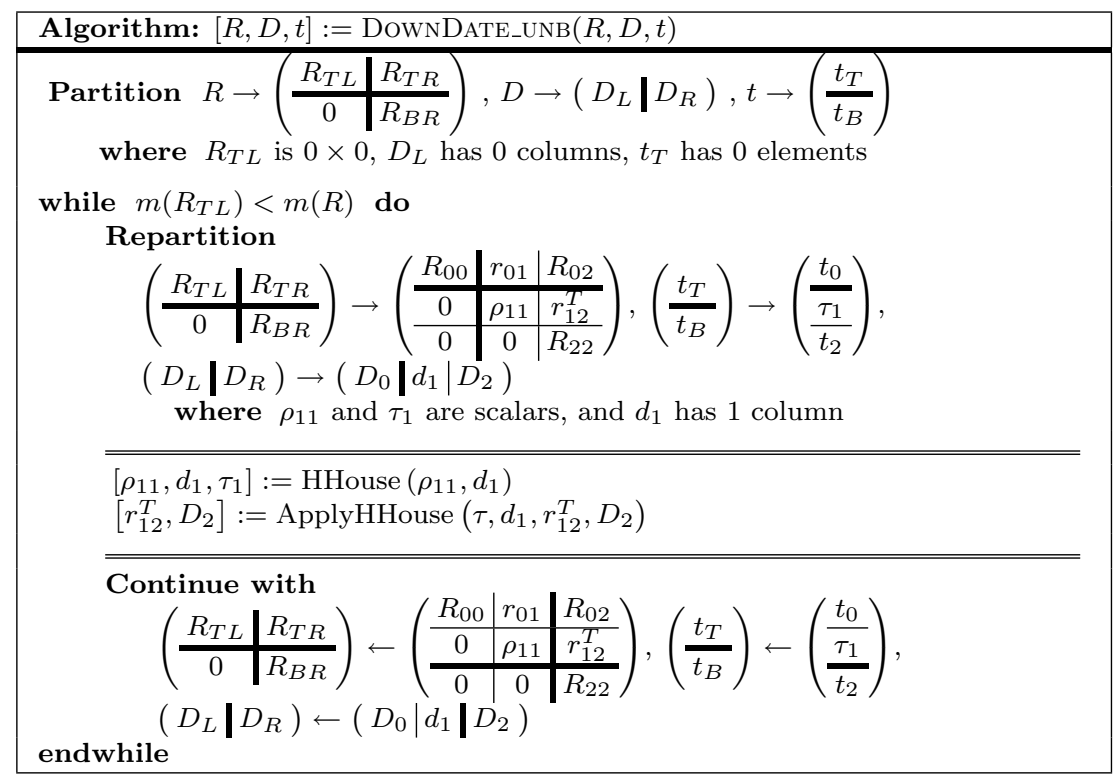

Fig. 2. Unblocked algorithm for downdating.

matrices contain

$$
\left(\begin{array}{c}
R \\
\hline \bar{D}
\end{array}\right)=\left(\begin{array}{c|c|c}
R_{00} & r_{01} & R_{02} \\
\hline 0 & \rho & r_{12}^{T} \\
\hline 0 & 0 & R_{22} \\
\hline 0 & d_{1} & D_{2}
\end{array}\right) .
$$

In the current step of the algorithm, a hyperbolic Householder Transformation is computed and applied so that

$$
\begin{aligned}
\left(\left(\begin{array}{c|c|c|c}
I & 0 & 0 & 0 \\
\hline 0 & 1 & 0 & 0 \\
\hline 0 & 0 & I & 0 \\
\hline 0 & 0 & 0 & I
\end{array}\right)-\frac{1}{\tau}\left(\begin{array}{c|c|c|c}
I & 0 & 0 & 0 \\
\hline 0 & 1 & 0 & 0 \\
\hline 0 & 0 & I & 0 \\
\hline 0 & 0 & 0 & -I
\end{array}\right)\left(\begin{array}{c}
0 \\
\hline \frac{1}{0} \\
\hline \overline{v_{1}}
\end{array}\right)\left(\begin{array}{c}
0 \\
\hline 1 \\
\hline 0 \\
\hline \overline{v_{1}}
\end{array}\right)^{H}\right)^{H}=\left(\begin{array}{c|c|c}
R_{00} & r_{01} & R_{02} \\
\hline 0 & \rho & r_{12}^{T} \\
\hline 0 & 0 & R_{22} \\
\hline \hline 0 & d_{1} & D_{2}
\end{array}\right) \\
=\left(\begin{array}{c|c|c|c}
R_{00} & r_{01} & R_{02} \\
\hline 0 & \tilde{\rho} & \tilde{r}_{12}^{T} \\
\hline 0 & 0 & R_{22} \\
\hline \hline 0 & 0 & D_{2}
\end{array}\right) .
\end{aligned}
$$

The hyperbolic Householder transformation associated with vector $v_{1}$ annihilates $d_{1}$, and thus the algorithm may simply set $d_{1}$ to zero (or, more practically, it may overwrite $d_{1}$ with $v_{1}$ to reduce storage). We must then use $\tau$ and $v_{1}$ to apply the hyperbolic Householder transformation to $r_{12}^{T}$ and $D_{2}$. Let us define the function ApplyHHouse for this purpose:

$$
\left[\tilde{r}_{12}^{T}, \tilde{D}_{2}\right]:=\text { ApplyHHouse }\left(\tau_{1}, v_{1}, r_{12}^{T}, D_{2}\right) \text {. }
$$

ACM Transactions on Mathematical Software, Vol. V, No. N, Month 20YY. 
An algorithm based on these steps is given in Figure 2.

\section{UP-AND-DOWNDATING}

Finally, let us consider the general problem where $A=\left(\frac{\frac{B}{\bar{C}}}{\overline{\bar{D}}}\right)$ with $A^{H} A=B^{H} B+$ $C^{H} C+D^{H} D$, where $C \in \mathbb{C}^{m_{C} \times n}, D \in \mathbb{C}^{m_{D} \times n}$. Let $R \in \mathbb{C}^{n \times n}$ be the Cholesky factor of $B^{H} B+D^{H} D$. The final question becomes how to compute the Cholesky factor of $B^{H} B+C^{H} C, \tilde{R}$, from $R, C$, and $D$. Clearly, one can do so by first updating and then downdating, or vise versa. We will develop an algorithm that does so in one step rather than two. We will call this the up-and-downdating problem.

We know that

$$
\begin{aligned}
\tilde{R}^{H} \tilde{R} & =B^{H} B+C^{H} C \\
& =R^{H} R+C^{H} C-D^{H} D \\
& =\left(\begin{array}{c}
R \\
\hline \bar{C} \\
\hline \bar{D}
\end{array}\right)\left(\begin{array}{c|c||c}
I_{n} & 0 & 0 \\
\hline \hline 0 & I_{m_{C}} & 0 \\
\hline \hline 0 & 0 & 0
\end{array}\right)\left(\begin{array}{c}
R \\
\hline \bar{C} \\
\hline \bar{D}
\end{array}\right) .
\end{aligned}
$$

In the remainder of this section, we will define

$$
\Sigma_{n, m, k}=\left(\begin{array}{c||c||c}
I_{n} & 0 & 0 \\
\hline \hline 0 & I_{m} & 0 \\
\hline \hline 0 & 0 & -I_{k}
\end{array}\right)
$$

The goal is going to be to compute a sequence of transformations, $\left\{G_{0}, G_{1}, \cdots, G_{n-1}\right\}$ such that

$$
G_{j} \Sigma_{n, m_{C}, m_{D}} G_{j}^{H}=\Sigma_{n, m_{C}, m_{D}}
$$

and

$$
\begin{aligned}
& B^{H} B+C^{H} C=\left(\begin{array}{c}
R \\
\hline \bar{C} \\
\hline \bar{D}
\end{array}\right)^{H}\left(\begin{array}{c||c||c}
I_{n} & 0 & 0 \\
\hline \hline 0 & I_{m_{C}} & 0 \\
\hline \hline 0 & 0 & -I_{m_{D}}
\end{array}\right)\left(\begin{array}{c}
R \\
\hline \overline{\bar{C}} \\
\hline \overline{\bar{D}}
\end{array}\right) \\
& =\left(\begin{array}{c}
\frac{R}{\bar{C}} \\
\hline \bar{D}
\end{array}\right)^{H} G_{0} \cdots G_{n-1}\left(\begin{array}{c||c||c}
I_{n} & 0 & 0 \\
\hline \hline 0 & I_{m_{C}} & 0 \\
\hline \hline 0 & 0 & -I_{m_{D}}
\end{array}\right) G_{n-1}^{H} \cdots G_{0}^{H}\left(\begin{array}{c}
\frac{R}{\bar{C}} \\
\hline \bar{D}
\end{array}\right) \\
& =\left(\begin{array}{c}
\tilde{R} \\
\hline 0
\end{array}\right)^{H}\left(\begin{array}{c||c||c}
I_{n} & 0 & 0 \\
\hline \hline 0 & I_{m_{C}} & 0 \\
\hline \hline 0 & 0 & -I_{m_{D}}
\end{array}\right)\left(\frac{\tilde{R}}{\overline{0}}\right)=\tilde{R}^{H} \tilde{R}
\end{aligned}
$$

In other words, given matrix $\left(\frac{\frac{R}{\bar{C}}}{\frac{\bar{D}}{\bar{D}}}\right)$ where $R$ is an $n \times n$ upper triangular matrix, ACM Transactions on Mathematical Software, Vol. V, No. N, Month 20YY. 
we would like to compute $\left\{G_{0}, \cdots, G_{n-1}\right\}$ so that

$$
G_{n-1}^{H} \cdots G_{0}^{H}\left(\frac{\frac{R}{\bar{C}}}{\overline{\bar{D}}}\right)=\left(\begin{array}{c}
\frac{\tilde{R}}{\overline{0}} \\
\overline{\overline{0}}
\end{array}\right)
$$

and

$$
G_{j}^{H}\left(\begin{array}{c||c||c}
I_{n} & 0 & 0 \\
\hline \hline 0 & I_{m_{C}} & 0 \\
\hline \hline 0 & 0 & -I_{m_{D}}
\end{array}\right) G_{j}=\left(\begin{array}{c|c||c|c}
I_{n} & 0 & 0 \\
\hline \hline 0 & I_{m_{C}} & 0 \\
\hline \hline 0 & 0 & -I_{m_{D}}
\end{array}\right) .
$$

Definition 9. Let $x=\left(\frac{\chi_{0}}{\frac{x_{1}}{y_{1}}}\right)$ with $\chi_{0} \in \mathbb{R}, x_{1} \in \mathbb{C}^{m_{C}}$ and $y_{1} \in \mathbb{C}^{m_{D}}$ be such that $x^{H} \Sigma_{1, m_{C}, m_{D}} x=\left|\chi_{0}\right|^{2}+x_{1}^{H} x_{1}-y_{1}^{H} y_{1} \neq 0$. We define the function

$$
\left[\tilde{\chi_{0}}, u_{1}, v_{1}, \tau\right]:=\operatorname{UDHouse}\left(\chi_{0}, x_{1}, y_{1}\right)
$$

so that

$$
\left[\left(\begin{array}{ccc}
1 & 0 & 0 \\
0 & I_{m_{C}} & 0 \\
0 & 0 & I_{m_{D}}
\end{array}\right)-\frac{1}{\tau}\left(\begin{array}{ccc}
1 & 0 & 0 \\
0 & I_{m_{C}} & 0 \\
0 & 0 & -I_{m_{D}}
\end{array}\right)\left(\begin{array}{c}
1 \\
u_{1} \\
v_{1}
\end{array}\right)\left(\begin{array}{c}
1 \\
u_{1} \\
v_{1}
\end{array}\right)^{H}\right]\left(\begin{array}{c}
\chi_{0} \\
x_{1} \\
y_{1}
\end{array}\right)=\left(\begin{array}{c}
\hat{\chi}_{0} \\
0 \\
0
\end{array}\right),
$$

where $\tau=\frac{1+u_{1}^{H} u_{1}-v_{1}^{H} v_{1}}{2}$.

We recognize this as the special case of the Generalized Householder Transformation where $\Sigma=\Sigma_{n, m_{C}, m_{D}}$.

Given the function UDHouse, we now wish to construct an algorithm that, given the Cholesky factor $R$ of $B^{H} B+D^{H} D$, computes the Cholesky factor of $B^{H} B+C^{H} C$. Here is the basic idea: Assume that the computation has progressed so that the matrices contain

$$
\left(\begin{array}{c}
R \\
\overline{\bar{C}} \\
\hline \bar{D}
\end{array}\right)=\left(\begin{array}{c|c|c}
R_{00} & r_{01} & R_{02} \\
\hline 0 & \rho_{11} & r_{12}^{T} \\
\hline 0 & 0 & R_{22} \\
\hline \hline 0 & c_{1} & C_{2} \\
\hline \hline 0 & d_{1} & D_{2}
\end{array}\right) .
$$

In the current step of the algorithm, an up-and-downdating Householder Transformation is computed and applied so that

$$
\left[\left(\begin{array}{c|c|c|c||c|}
I & 0 & 0 & 0 & 0 \\
\hline 0 & 1 & 0 & 0 & 0 \\
\hline 0 & 0 & I & 0 & 0 \\
\hline 0 & 0 & 0 & I & 0 \\
\hline \hline 0 & 0 & 0 & 0 & I
\end{array}\right)-\frac{1}{\tau}\left(\begin{array}{c|c|c|c|c|c}
I & 0 & 0 & 0 & 0 \\
\hline 0 & 1 & 0 & 0 & 0 \\
\hline 0 & 0 & I & 0 & 0 \\
\hline \hline 0 & 0 & 0 & I & 0 \\
\hline \hline 0 & 0 & 0 & 0 & -I
\end{array}\right)\left(\begin{array}{c}
0 \\
\hline \frac{1}{0} \\
\hline 0 \\
\hline \overline{u_{1}} \\
\hline \overline{v_{1}}
\end{array}\right)\left(\begin{array}{c}
0 \\
\hline 1 \\
\hline 0 \\
\hline \overline{u_{1}} \\
\hline \overline{v_{1}}
\end{array}\right)^{H}\right]\left(\begin{array}{c|c|c|c|}
R_{00} & r_{01} & R_{02} \\
\hline 0 & \rho_{11} & r_{12}^{T} \\
\hline 0 & 0 & R_{22} \\
\hline 0 & c_{1} & C_{2} \\
\hline \hline 0 & d_{1} & D_{2}
\end{array}\right)
$$

ACM Transactions on Mathematical Software, Vol. V, No. N, Month 20YY. 


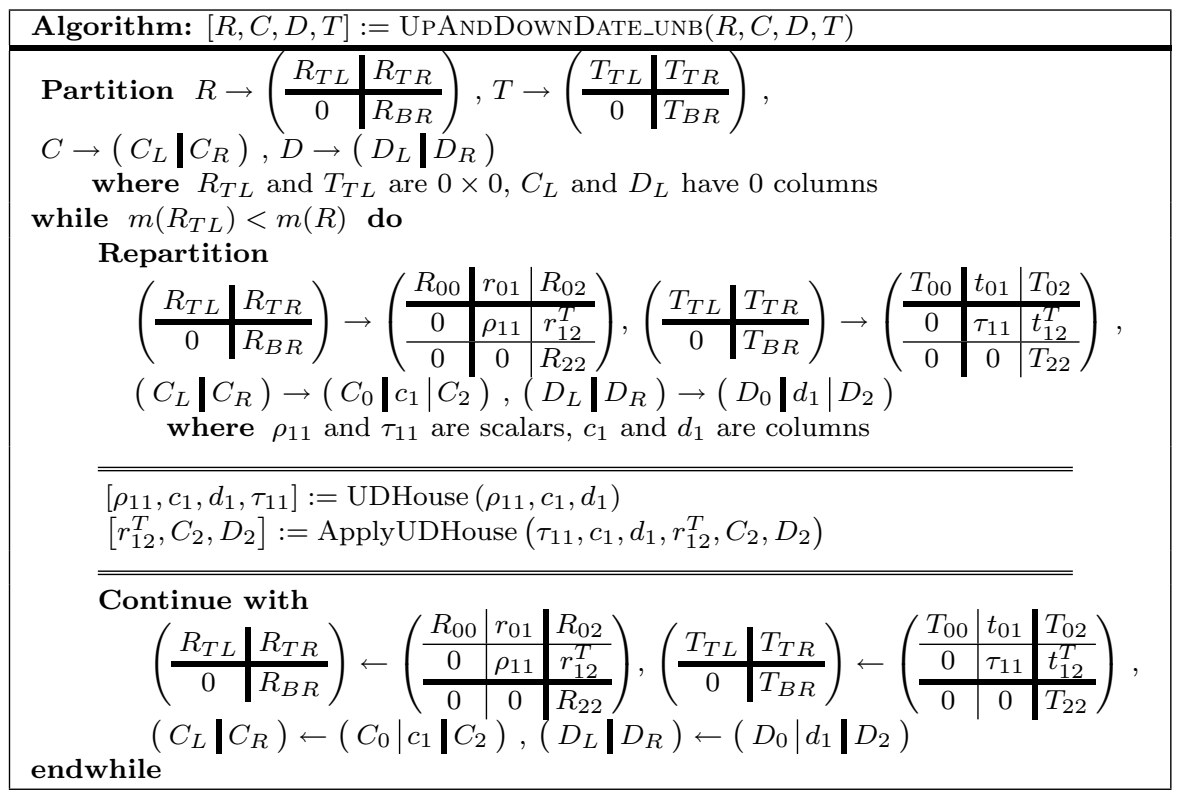

Fig. 3. Unblocked algorithm for up-and-downdating.

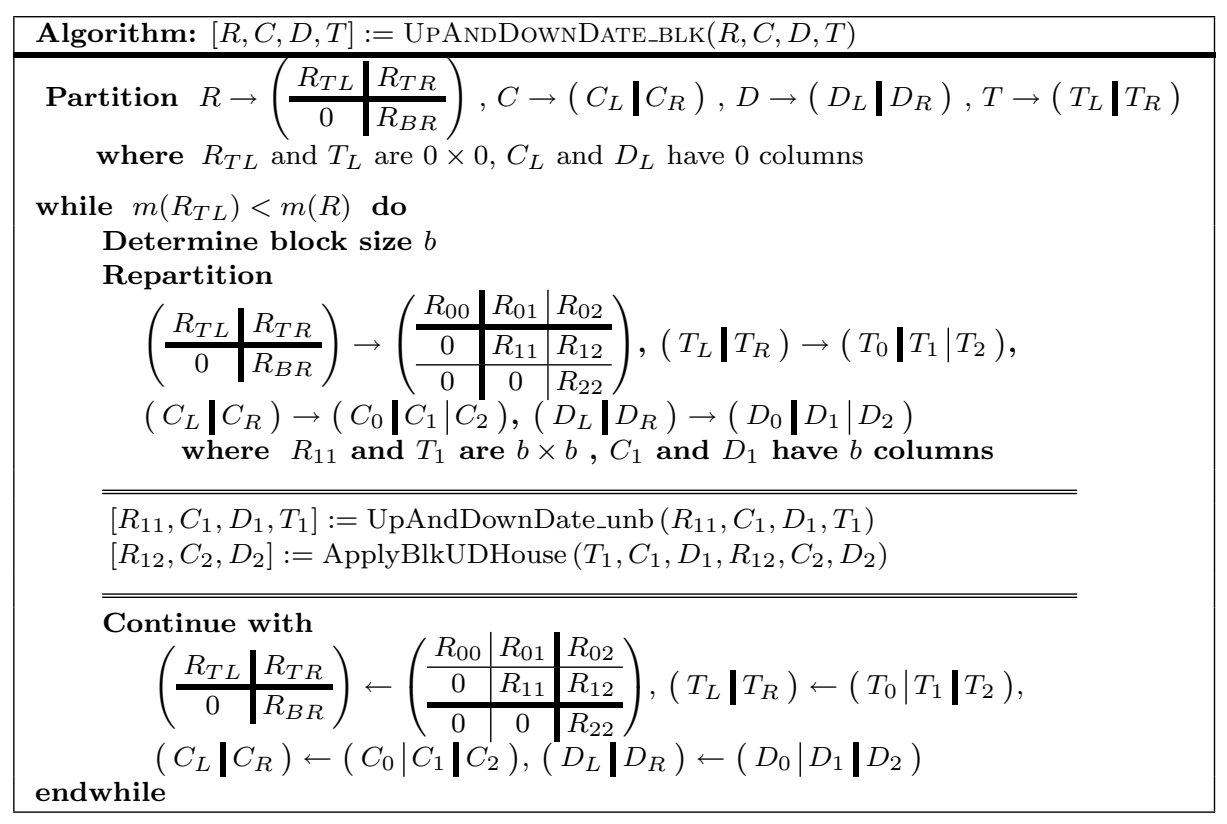

Fig. 4. Blocked algorithm for up-and-downdating. 


$$
=\left(\begin{array}{c|c|c}
R_{00} & r_{01} & R_{02} \\
\hline 0 & \tilde{\rho}_{11} & \tilde{r}_{12}^{T} \\
\hline 0 & 0 & R_{22} \\
\hline \hline 0 & 0 & \tilde{C}_{2} \\
\hline \hline 0 & 0 & \tilde{D}_{2}
\end{array}\right) .
$$

The up-and-downdating Householder transformation annihilates $c_{1}$ and $d_{1}$, and thus the algorithm may simply set these vectors to zero (or, more practically, it may overwrite $c_{1}$ and $d_{1}$ with $u_{1}$ and $v_{1}$, respectively, to reduce storage). We must then use $\tau, u_{1}$, and $v_{1}$ to apply the up-and-downdating Householder transformation to $r_{12}^{T}, C_{2}$, and $D_{2}$. Let us define the function APPLYUDHouse for this purpose:

$$
\left[\tilde{r}_{12}^{T}, \tilde{C}_{2}, \tilde{D}_{2}\right]:=\text { ApplyUDHouse }\left(\tau_{1}, u_{1}, v_{1}, r_{12}^{T}, C_{2}, D_{2}\right) .
$$

An algorithm based on these steps is given in Figure 3.

Note that in the previous two sections, we only discuss and illustrate unblocked algorithms. We now move to discuss a blocked algorithm for up-and-downdating, which is given in Figure 4. In this algorithm, the statement

$$
\left[R_{12}, C_{2}, D_{2}\right]:=\text { ApplyBlkUDHouse }\left(T_{1}, C_{1}, D_{1}, R_{12}, C_{2}, D_{2}\right)
$$

performs the update

$$
\left.\left(\begin{array}{c}
\tilde{R}_{12} \\
\hline \bar{C}_{2} \\
\hline \hline \tilde{D}_{2}
\end{array}\right):=\left[\begin{array}{c|c||c}
I & 0 & 0 \\
\hline \hline 0 & I & 0 \\
\hline \hline 0 & 0 & I
\end{array}\right)-\left(\begin{array}{c|c||c}
I & 0 & 0 \\
\hline \hline 0 & I & 0 \\
\hline \hline 0 & 0 & -I
\end{array}\right)\left(\begin{array}{c}
I \\
\hline \overline{C_{1}} \\
\hline \overline{D_{1}}
\end{array}\right) T_{1}^{-T}\left(\begin{array}{c}
I \\
\hline \overline{C_{1}} \\
\hline \overline{D_{1}}
\end{array}\right)^{H}\right]\left(\begin{array}{c}
\frac{R_{12}}{\overline{C_{2}}} \\
\hline \overline{D_{2}}
\end{array}\right)
$$

where $T_{1}=\operatorname{striu}\left(I+C_{1}^{H} C_{1}-D_{1}^{H} D_{1}\right)+\frac{1}{2} \operatorname{diag}\left(I+C_{1}^{H} C_{1}-D_{1}^{H} D_{1}\right)$. The submatrix $T_{1}$ may be computed via the following steps ${ }^{45}$ :

$$
\begin{aligned}
& T_{1}:=\operatorname{triu}\left(I+C_{1}^{H} C_{1}-D_{1}^{H} D_{1}\right) \\
& T_{1}:=\text { ScaleDiagonal }\left(\frac{1}{2}, T_{1}\right)
\end{aligned}
$$

where the ScaleDiagonal operation scales the diagonal of the second argument by the first argument. With $T_{1}$ computed, we may perform the update as follows:

$$
\begin{aligned}
W & :=T_{1}^{-H}\left(R_{12}+C_{1}^{H} C_{2}+D_{1}^{H} D_{2}\right) \\
\left(\frac{\tilde{R}_{12}}{\overline{\tilde{C}_{2}}}\right) & :=\left(\frac{R_{12}}{\overline{\frac{C_{2}}{\tilde{D}_{2}}}}\right)-\left(\frac{\frac{I}{\overline{D_{2}}}}{\overline{\frac{C_{1}}{-D_{1}}}}\right) W
\end{aligned}
$$

This blocked algorithm captures the blocked algorithms for updating and downdating, since $D$ or $C$ can be taken to be "empty".

\footnotetext{
${ }^{4}$ In practice, we find it most convenient to compute $T_{1}$ within the unblocked algorithm for upand-downdating, UpAndDownDATE_UnB. Note that we omit this step from the algorithm shown in Figure 3 and instead only show the storing of the $\tau$ values along the diagonal of $T_{1}$.

${ }^{5}$ Note that $\operatorname{triu}(A)$ returns a matrix consisting of the upper triangular part of $A$ with zeros elsewhere.
}

ACM Transactions on Mathematical Software, Vol. V, No. N, Month 20YY. 


\section{SOLVING A SYSTEM}

Consider the matrices $B \in m_{B} \times n, C \in m_{C} \times n$, and $D \in m_{D} \times n$. The upand-downdating problem starts with a matrix $R$ such that $B^{H} B+C^{H} C=R^{H} R$. Where does this come from? Typically, it comes from solving the linear leastsquares problem

$$
\min _{x}\left\|\left(\begin{array}{c}
B \\
D
\end{array}\right) x-\left(\begin{array}{c}
b_{B} \\
b_{D}
\end{array}\right)\right\| .
$$

There are two standard ways of solving this problem: normal equations and QR factorization (via Householder transformations). Since we are using Generalized Householder transformations to updowndate, we restrict ourselves to the case where the original $R$ came from (the equivalent of) a $\mathrm{QR}$ factorization.

So, we assume that we have computed (the equivalent of) the QR factorization

$$
\left(\begin{array}{l}
B \\
D
\end{array}\right)=Q R
$$

and then solved

$$
R x=Q^{H}\left(\begin{array}{l}
b_{B} \\
b_{D}
\end{array}\right)=b_{B D}
$$

Now, after the updowndate step, what one is interested in is solving

$$
\min _{\tilde{x}}\left\|\left(\begin{array}{c}
B \\
C
\end{array}\right) \tilde{x}-\left(\begin{array}{c}
b_{B} \\
b_{C}
\end{array}\right)\right\|
$$

which could be solved via the QR factorization

$$
\left(\begin{array}{c}
B \\
C
\end{array}\right)=\tilde{Q} \tilde{R}
$$

and then solved

$$
\tilde{R} \tilde{x}=\tilde{Q}^{H}\left(\begin{array}{l}
b_{B} \\
b_{C}
\end{array}\right)=b_{B C} .
$$

Now, we have computed up-and-downdating Householder transformations $G_{j}$ so that

$$
\left(\begin{array}{c}
\frac{\tilde{R}}{\overline{0}} \\
\overline{\overline{0}}
\end{array}\right)=G_{n-1}^{H} \cdots G_{0}^{H}\left(\frac{\frac{R}{\bar{C}}}{\overline{\bar{D}}}\right)
$$

Note that

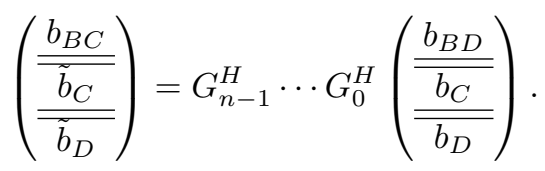

Thus, applying the block up-and-downdating Householder transformations from the left will up-and-downdate $b_{B D}$ into $b_{B C}$. This computation may be performed via the same APPLYBLKUDHOusE operation described in the previous section and 
used in Figure 4:

$$
\begin{aligned}
{[\tilde{R}, \tilde{C}, \tilde{D}, \tilde{T}] } & :=\operatorname{UpAndDownDate\_ Blk}(R, C, D, T) \\
{\left[b_{B C}, \tilde{b}_{C}, \tilde{b}_{D}\right] } & :=\operatorname{ApplyBlkUDHouse}\left(\tilde{T}, \tilde{C}, \tilde{D}, b_{B D}, b_{C}, b_{D}\right)
\end{aligned}
$$

At this point, $R$ and $b_{B D}$ have been up-and-downdated to $\tilde{R}$ and $b_{B C}$, respectively, and so a new solution to the system may be computed by solving

$$
\tilde{R} \tilde{x}=b_{B C} .
$$

\section{AN ALGORITHM-BY-BLOCKS}

As part of the FLAME project, we have developed and reported on algorithm-byblocks for various linear algebra operations and how to schedule them to distributed memory as well as multithreaded parallel architectures. An algorithm-by-blocks views a matrix as a collection of submatrices (blocks), possibly hierarchically. Each block becomes a unit of data and each computation with a block becomes a unit of computation.

- In [Quintana-Ortí and van de Geijn 2008; Gunter and van de Geijn 2005] we give algorithms-by-tiles for out-of-core $\mathrm{LU}$ and $\mathrm{QR}$ factorization. A tile is a block that corresponds to a unit for I/O. By modifying the pivoting strategy for LU factorization and the computation of Householder transformations for QR factorization, the computation can be cast in terms of operations with blocks while only increasing the operation count by a lower order term.

-In [Chan et al. 2007] a runtime system, SuperMatrix, for scheduling algorithmby-blocks to multiple threads is introduced. Implementations of algorithms-byblocks utilizing this runtime system are discussed in a large number of conference papers and summarized in a journal paper [Quintana-Ortí et al. 2009]. The idea is that the algorithm-by-blocks generates a Directed Acyclic Graph (DAG) of operations and dependencies which are then scheduled for execution by threads at runtime.

The effort focuses on solving the programmability problem: algorithms are coded in a style that closely resembles the algorithms in the figures in this paper. The algorithm-by-blocks is coded in a very similar style. By separating the generation of the DAG by the algorithm from the scheduling of that DAG, the library routine needs not change when the scheduling policy is modified.

Details of the algorithm-by-blocks for up-and-downdating are essentially identical to those of the updating algorithm-by-tiles in [Gunter and van de Geijn 2005] and the QR factorization algorithm-by-blocks scheduled with SuperMatrix in [Quintana-Ortí et al. 2008] or PLASMA in [Buttari et al. 2008], except that minor modifications are made when computing with the matrix that is removed as part of the downdating. Thus, we don't give further details here and merely report performance, in the next section.

\section{PERFORMANCE}

In this section, we provide performance results for various implementations of the up-and-downdating algorithm, including a high-performance algorithm-by-blocks.

ACM Transactions on Mathematical Software, Vol. V, No. N, Month 20YY. 
All experiments were performed using double-precision floating-point arithmetic on a Dell PowerEdge R900 server consisting of four Intel "Dunnington" six-core processors, providing a total of 24 cores with a combined peak performance of 255 GFLOPs $\left(255 \times 10^{9}\right.$ floating-point operations per second) with 96 GBytes of shared main memory. Performance experiments were gathered under the GNU/Linux 2.6.18 operating system. Source code was compiled by the Intel $\mathrm{C} / \mathrm{C}++$ Compiler, version 11.1.

In addition to reporting performance for the up-and-downdating operation, for comparison we also provide performance data for QR factorization via the UT transform, as the two operations are closely related. We report performance for the following implementations in Figures 5 and 6:

-UDDUT. A sequential implementation of the blocked algorithm for the up-anddowndating operation shown in Figure 4.

- QRUT. A sequential implementation of a blocked algorithm for a QR factorization via the UT transform [Joffrain et al. 2006].

-UdDUTABB. A multithreaded implementation of an algorithm-by-blocks for the up-and-downdating operation.

- QRUTABB. A multithreaded implementation of an algorithm-by-blocks for a QR factorization via the UT transform [Quintana-Ortí et al. 2007].

- sequential dgeqrf. A sequential implementation of the LAPACK QR factorization routine.

- multithreaded dgeqrf. A multithreaded implementation of the LAPACK QR factorization routine.

These implementations were timed in two ways: linked to a sequential build of GotoBLAS2 1.10 and linked to sequential build of Intel's MKL 10.2.2. The dgeqrf implementations, likewise, were obtained from both GotoBLAS2 1.10 and MKL 10.2.2. Parallelism was obtained from the UDDUTABB and QRUTABB via the SuperMatrix runtime system [Chan et al. 2007; Chan et al. 2007]. For completeness, we also report performance of the netlib implementation of dgeqrf (modified to use a larger block size) when linked to multithreaded GotoBLAS and MKL.

Performance results are computed using an operation count of $2 n^{2}\left(m_{C}+m_{D}\right)$ for the up-and-downdate operation and $2 n^{2}\left(m-\frac{n}{3}\right)$ for a QR factorization. This counts useful operations, ignoring extra operations that are performed so that the blocked algorithms can cast computation in terms of matrix-matrix multiplication. The $y$-axes of the graphs are scaled to indicate the peak performance for the number of cores utilized.

Matrix dimensions for QR factorization were carefully chosen so that the floatingpoint operation count would closely resemble that of the up-and-downdating operation. Specifically, since we performed up-and-downdating experiments where $m_{C}=m_{D}=n$, we chose the matrix dimensions for QR factorization to be $m=3 n$.

In Figure 5 (top) we report the performance of the blocked algorithms using a single core, choosing the block size equal to 64 . The rates of computation achieved by the up-and-downdating algorithms is better than those achieved by the QR factorization because more computation is cast in terms of matrix-matrix multiplication. Timings for the same blocked algorithms, in addition to netlib dgeqrf linked to 

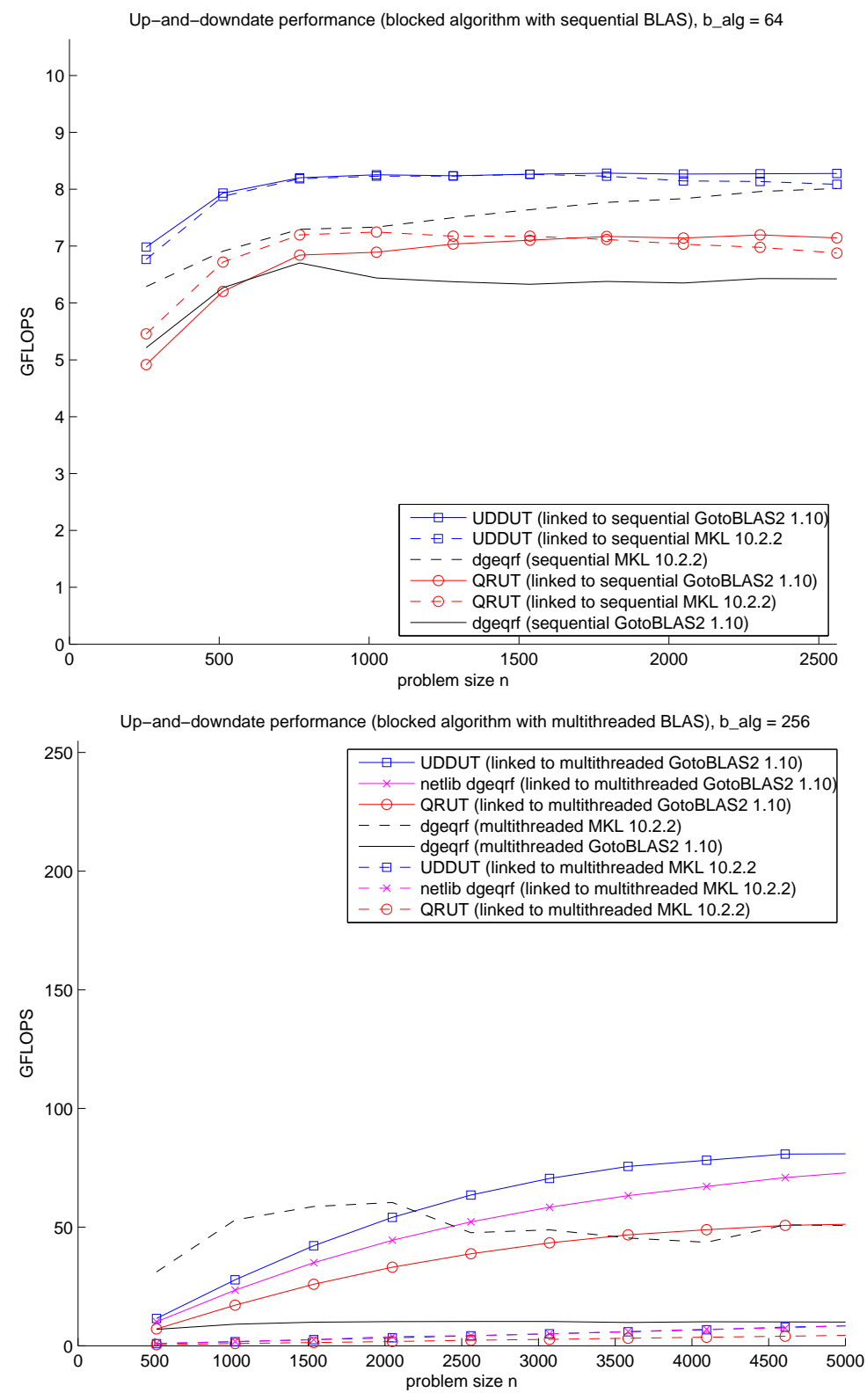

Fig. 5. Performance of sequential and multithreaded implementations of the up-and-downdating operation. Top: Sequential up-and-downdating implementations compared to various sequential QR factorizations, using an algorithmic block size of 64. Bottom: Blocked algorithm for up-anddowndating linked to multithreaded BLAS compared to various multithreaded QR factorizations, with an algorithm block size of 256. Multithreaded performance was gathered on a 24 core system using 24 threads. QR factorization experiments were performed on $m \times n$ matrices where $m=3 n$ while up-and-downdating experiments reflect $m_{C}=m_{D}=n$. Note that legend entries are sorted according to performance at largest problem size.

ACM Transactions on Mathematical Software, Vol. V, No. N, Month 20YY. 

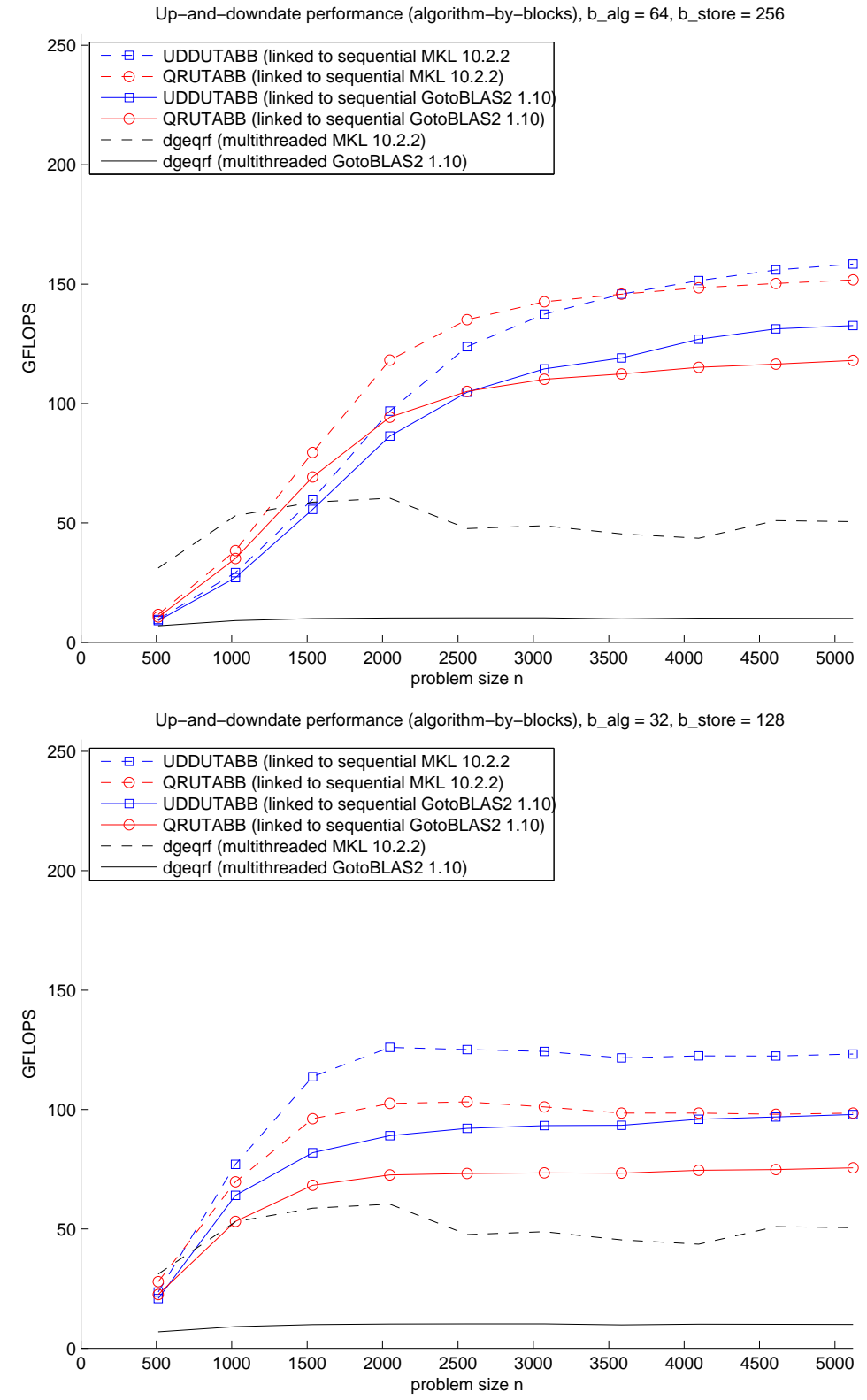

Fig. 6. Multithreaded algorithm-by-blocks for up-and-downdating compared to various multithreaded QR factorizations, including the multithreaded QR factorization in MKL. Algorithmic and storage block sizes are chosen to equal 64 and 256, respectively, in the top graph and 32 and 128 in the bottom graph. Performance was gathered on a 24 core system using 24 threads. QR factorization experiments were performed on $m \times n$ matrices where $m=3 n$ while up-anddowndating experiments reflect $m_{C}=m_{D}=n$. Note that legend entries are sorted according to performance at largest problem size. 
multithreaded BLAS, using 24 cores and an algorithmic block size of 256 are given in Figure 5 (bottom). We note that while MKL's dgeqrf achieves very good performance, our implementation of the QR factorization and the up-and-downdating algorithm does not when linked to MKL's multithreaded BLAS. This is likely due to how the matrix-matrix multiplication (dgemm) is parallelized. When linked to GotoBLAS2, the performance is much improved, although still well below peak and still lagging behind the modified netlib implementation of dgeqre.

In Figure 6 we report the performance of the algorithms-by-blocks. The ability to store matrices by blocks combined with a run-time system that schedules operations to threads greatly improves performance. When the storage block size $\left(b_{\text {store }}\right)$ and algorithmic block size $\left(b_{\text {alg }}\right)$ are relatively large, ramp-up is slow while the asymptotic performance is better.

\section{CONCLUSION}

In this paper, we have presented unblocked and blocked algorithms for the upand/or downdating problem. It has been shown that blocked algorithms can be easily formulated and that high performance can be achieved.

\section{Acknowledgements}

This research was partially sponsored by NSF grant CCF-0917167 and a grant from Microsoft. We would like to thank John Reid and the ananymous referees for many helpful suggestions.

Any opinions, findings and conclusions or recommendations expressed in this material are those of the author(s) and do not necessarily reflect the views of the National Science Foundation (NSF).

\section{REFERENCES}

Bischof, C. And VAn LoAn, C. 1987. The WY representation for products of Householder matrices. SIAM J. Sci. Stat. Comput. 8, 1 (Jan.), s2-s13.

Buttari, A., Langou, J., Kurzak, J., And Dongarra, J. 2008. Parallel tiled qr factorization for multicore architectures. Concurr. Comput. : Pract. Exper. 20, 13, 1573-1590.

Chan, E., Quintana-Ortí, E. S., Quintana-Ortí, G., and van de Geijn, R. 2007. SuperMatrix out-of-order scheduling of matrix operations for SMP and multi-core architectures. In SPAA '07: Proceedings of the Nineteenth ACM Symposium on Parallelism in Algorithms and Architectures. 116-126.

Chan, E., Van Zee, F. G., Bientinesi, P., Quintana-Ortí, E. S., Quintana-Ortí, G., And VAN DE GEIJN, R. 2007. Supermatrix: A multithreaded runtime scheduling system for algorithms-by-blocks. FLAME Working Note \#25 TR-07-41, The University of Texas at Austin, Department of Computer Sciences. August.

Dongarra, J. J., Du Croz, J., Hammarling, S., And Duff, I. 1990. A set of level 3 basic linear algebra subprograms. ACM Trans. Math. Soft. 16, 1 (March), 1-17.

Gunter, B. C. And van de Geijn, R. A. 2005. Parallel out-of-core computation and updating the QR factorization. ACM Transactions on Mathematical Software 31, 1 (March), 60-78.

Joffrain, T., Low, T. M., Quintana-Ortí, E. S., van de Geijn, R., and Van Zee, F. G. 2006. Accumulating householder transformations, revisited. ACM Trans. Math. Softw. 32, 2, $169-179$

PugLisi, C. 1992. Modification of the Householder method based on the compact wy representation. SIAM J. Sci. Stat. Comput. 13, 723-726.

Quintana-Ortí, E. S. And VAn DE Geijn, R. A. 2008. Updating an LU factorization with pivoting. ACM Trans. Math. Softw. 35, 2, 1-16.

ACM Transactions on Mathematical Software, Vol. V, No. N, Month 20YY. 
Quintana-Ortí, G., Quintana-Ortí, E. S., Chan, E., van de Geijn, R. A., and Van Zee, F. G. 2008. Scheduling of QR factorization algorithms on SMP and multi-core architectures. In PDP '08: Proceedings of the 16th Euromicro Conference on Parallel, Distributed and Network-Based Processing (PDP 2008). IEEE Computer Society, Washington, DC, USA, 301-310.

Quintana-Ortí, G., Quintana-Ortí, E. S., Chan, E., Van Zee, F. G., and van de Geijn, R. 2007. Scheduling of QR factorization algorithms on SMP and multi-core architectures. FLAME Working Note \#24 TR-07-37, The University of Texas at Austin, Department of Computer Sciences. July.

Quintana-Ortí, G., Quintana-Ortí, E. S., van de Geijn, R. A., Van Zee, F. G., and Chan, E. 2009. Programming matrix algorithms-by-blocks for thread-level parallelism. ACM Transactions on Mathematical Software 36, 3 (July), 14:1-14:26.

Schreiber, R. AND VAN LoAn, C. 1989. A storage-efficient WY representation for products of Householder transformations. SIAM J. Sci. Stat. Comput. 10, 1 (Jan.), 53-57.

Stewart, M. and Stewart, G. W. 1998. On hyperbolic triangularization: Stability and pivoting. SIAM Journal on Matrix Analysis and Applications 19, 4, 847-860.

Sun, X. 1996. Aggregations of elementary transformations. Tech. Rep. Technical report DUKETR-1996-03, Duke University.

WALKER, H. F. 1988. Implementation of the GMRES method using Householder transformations. SIAM J. Sci. Stat. Comput. 9, 1, 152-163.

YAN, W.-M. AND Chung, K.-L. 1997. A block representation for products of hyperbolic householder transform. Applied Mathematics Letters 10, 1 (January), 109-112.

Received Month Year; revised Month Year; accepted Month Year 